\title{
The Use of Quantitative Magnetic Resonance Perfusion for Assessment of CBF in The Perioperative Management of Carotid Stenosis: Case Illustra- tion
}

\author{
Joseph G. Adel, Arun K. Sherma, Timothy J. Carroll, Ziad A. Hage, Jeffery W. Miller, Matthew T. \\ Walker, H. Hunt Batjer and Bernard R. Bendok*
}

\begin{abstract}
Department of Neurological Surgery, Northwestern University Feinberg School of Medicine, Feinberg School of Medicine, 676 N. St. Clair, Suite 2210, Chicago, IL 60611, USA
\end{abstract}

\begin{abstract}
Objective and Importance: Internal Carotid Artery (ICA) stenosis is a common condition with a high prevalence in the normal population and carries a high risk of stroke. Hemodynamic impairment has been proven to be a powerful and independent risk factor for stroke. Understanding the hemodynamics beyond a stenosis may play a role in selecting patients who would benefit from treatment, determining the success of the treatment, and monitoring for disease recurrence. Our group has developed a unique quantitative approach to MR perfusion. We report on the use of Quantitative Magnetic Resonance Perfusion (MRP) for assessment of Cerebral Blood Flow (CBF) pre- and post- Carotid Angioplasty and Stenting for symptomatic ICA stenosis.

Clinical Presentation: A 71-year-old female with significant comorbidities presented with two episodes of left transient ischemic attacks (TIAs) over the course of one month related to high grade left ICA stenosis. Preoperative quantitative MRP showed asymmetry in CBF between the two hemispheres. Carotid angioplasty and stenting was recommended.

Intervention: Left ICA angioplasty and stenting was performed and reduced the stenosis to $<10 \%$. Quantitative MRP done after the procedure revealed improved CBF to the affected side and normalization in the asymmetry of perfusion between the two hemispheres. Quantitative MRP values showed that affected regions were hypoperfused, but flow was sufficiently maintained so as to avoid infarct.

Conclusion: Relative MRP images allowed us to elucidate improvement in perfusion resulting form carotid angioplasty and stenting. This novel quantitative MRP allows quantification of CBF and the potential to provide additional information on the degree of hemodynamic compromise.
\end{abstract}

Keywords: Carotid artery angioplasty and stenting, cerebral blood flow, intracranial atherosclerosis, magnetic resonance perfusion, quantitative magnetic resonance perfusion.

\section{INTRODUCTION}

Internal Carotid Artery (ICA) stenosis, caused by atherothrombotic deposition, is a common condition with a high prevalence in the normal population $(\sim 0.5 \%$ in patients over 65 -year-old and $\geq 10 \%$ between ages $75-85)$. The annual average risk of stroke for an asymptomatic stenosis varies between $1 \%$ and $3 \%$, and may reach up to $15 \%$ if the stenosis is symptomatic [1]. Stenosis of the extracranial ICA accounts for less than $20 \%$ of all strokes and that of the intracranial arteries alone or in combination with extracranial stenosis accounts for another $20 \%$ [1]. In the presence of stenosis, the reduction in the mean arterial pressure of the cortical arteries varies with the degree of stenosis and depends on the status of the collateral circulation [2]. Knowing that the Circle of Willis and/or the external to internal carotid artery collaterals may maintain the perfusion pressure beyond a stenosis makes it impossible to correlate a percent of

*Address correspondence to this author at the Department of Neurological Surgery, Northwestern University Feinberg School of Medicine, Feinberg School of Medicine, 676 N. St. Clair, Suite 2210, Chicago, IL 60611, USA; Tel: (312) 695-0464; Fax: (312) 695-0225; E-mail: bbendok@nmff.org asymptomatic stenosis (Example $>70 \%$ ) with hemodynamic impairment. Although most ischemic strokes secondary to ICA stenosis are attributed to embolic phenomena, the presence of hemodynamic impairment has been proven to be a powerful and independent risk factor for stroke [2-4]. For this reason, understanding the hemodynamics beyond a stenosis may play a role in selecting patients who would benefit from treatment, determining the success of the treatment, and monitoring for recurrence. Available techniques for assessing $\mathrm{CBF}$ include positron-emission tomography (PET), single-photon emission computed tomography (SPECT), and various Magnetic Resonance techniques that use relative quantification. We report on the use of Magnetic Resonance Perfusion (MRP) for assessment of CBF pre- and post-Carotid Angioplasty and Stenting (CAS) in a 71-yearold woman who presented with symptomatic left ICA stenosis. Quantitative MRP values were calculated using a recently developed technique [5,6].

\section{MATERIALS AND METHODOLOGY}

\section{Patient}

The patient is a 71-year-old female with a history of severe coronary artery disease and Chronic Obstructive Pul- 


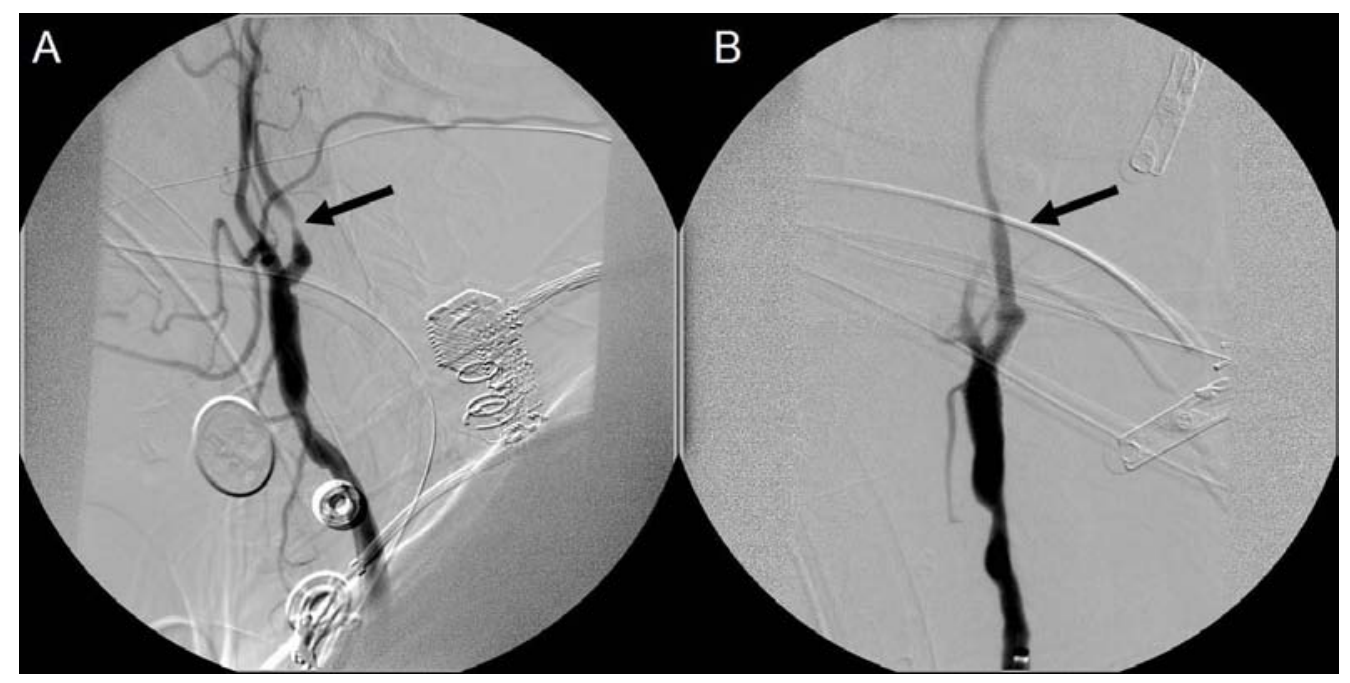

Fig. (1). (A) Pre-treatment lateral angiogram demonstrating a high- grade left ICA stenosis at the distal bulb. (B) Post- treatment lateral carotid artery angiogram.

monary Disease (COPD) who presented with two episodes of left hemisphere TIAs (transient right hand weakness, but no aphasic trends) over the course of one month related to high grade left carotid artery bifurcation stenosis (Fig. 1A). Carotid angiography revealed a $90 \%$ stenosis of the left ICA at the carotid artery bifurcation as well as $50 \%$ stenosis more proximally in the left common carotid artery. CAS was recommended and performed due to her co-morbidities. ICA angioplasty and stenting reduced the stenosis to $<10 \%$ (Fig. 1B). The more proximal stenosis was not treated. No complications occurred due to the procedure. Quantitative MR perfusion was performed prior to treatment (Fig. 2) as well as after treatment (Fig. 3). At 21 months, the patient denied any TIAs and was neurologically intact. Follow-up carotid duplex showed no re-stenosis. At one month follow-up the patient denied any TIAs and was neurologically intact.

\section{MRI Studies}

Quantitative CBF (qCBF) images were acquired preoperatively using the standard stroke imaging in our insti- tute. This includes T1, T2, FLAIR, Time Of Flight (TOF) and perfusion/diffusion weighted images. Preoperatively the perfusion scans were acquired using a recently reported MRI technique [7] which quantifies cerebral perfusion based on a simple modification of the standard deconvolution analysis $[5,6,8]$. Preoperative assessment of qCBF acquired a standard gradient recalled echo planar imaging (GE-EPI), MRI perfusion (TR/TE $=1440 \mathrm{~ms} / 47 \mathrm{~ms}, 12,5.0 \mathrm{~mm}$ slices) with a $20 \mathrm{ml}$ injection of contrast agent. Post- operatively an additional contrast enhanced MR angiogram was acquired (3D FLASH, FOV $=310 \mathrm{~mm} \times 251 \mathrm{~mm}$, matrix $=512 \times 416$, BW=490 Hz/pixel, $801.0 \mathrm{~mm}$ slices, TR/TE/F.A.=3.36 $\mathrm{ms} / 1.18 \mathrm{~ms} / 25^{\circ}$, contrast $=20 \mathrm{ml}$ GD-DTPA). The additional contrast injection served as a preloading contrast dose that allowed the acquisition of a slightly modified MR qCBF scan. In this case, a single shot spin-echo EPI (TR/TE $=1500$ $\mathrm{ms} / 72 \mathrm{~ms}, 13,5.0 \mathrm{~mm}$ slices) was acquired in combination with an 8-channel head coil and parallel imaging technique (iPAT, 2X acceleration). Spin-echo perfusion images have been shown to be less prone to inhomogeniety artifact than
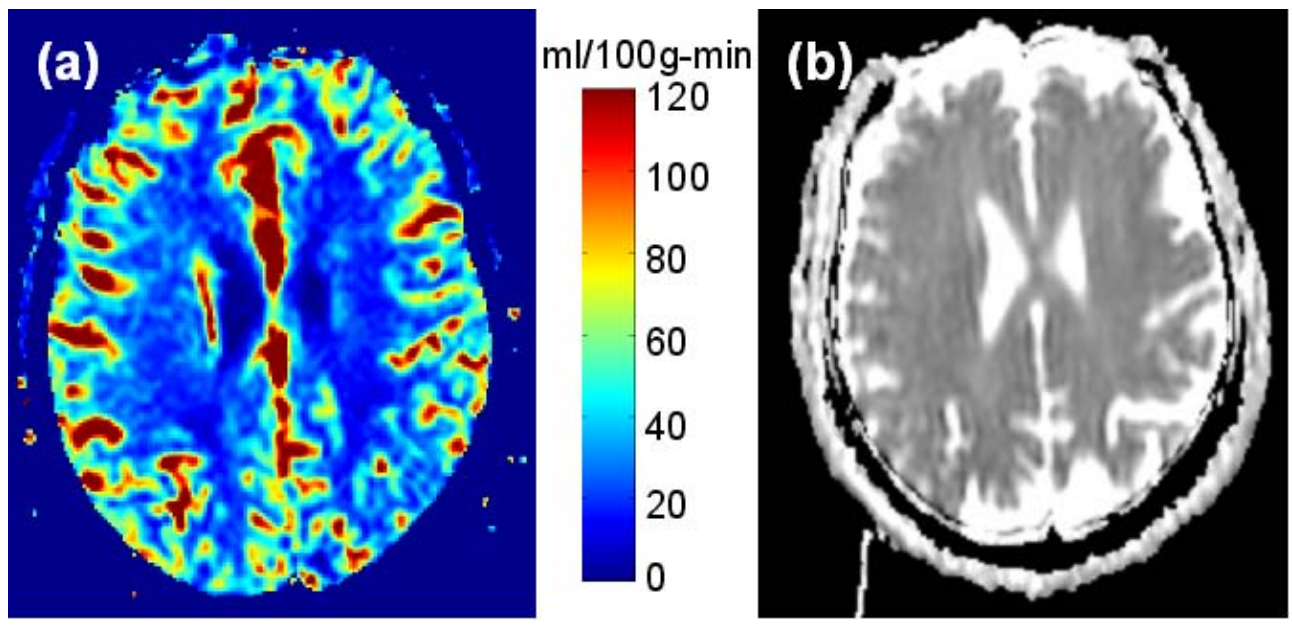

Fig. (2). Pre- operative (a) quantitative Cerebral Blood Flow (qCBF) and (b) Apparent Diffusion Coefficient (ADC) images. Note the asymmetry in white matter qCBF values (right= $24.42 \pm 9.41 \mathrm{~mL} / 100 \mathrm{~g}$ - $\mathrm{min}$; left= $19.53 \pm 5.76 \mathrm{~mL} / 100 \mathrm{~g}$ - min). The slight left hemisphere hypoperfusion did not result in reduced ADC. 

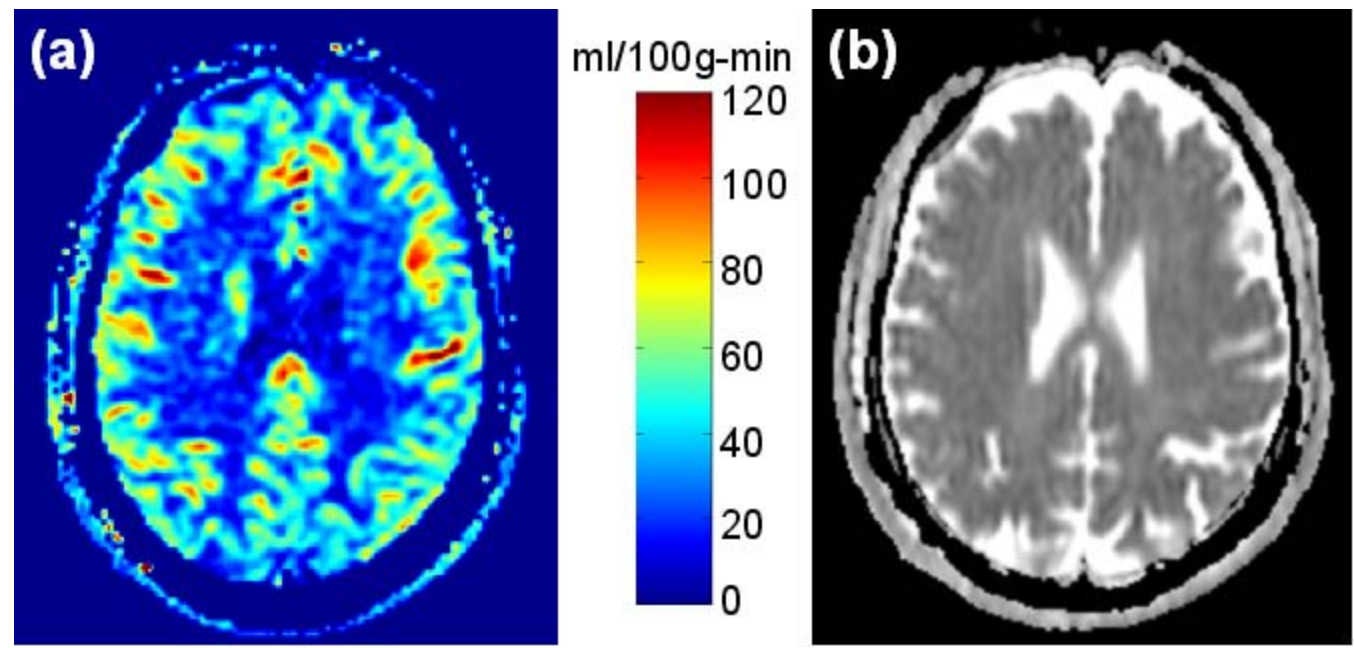

Fig. (3). Post- operative (a) quantitative Cerebral Blood Flow (qCBF) and (b) Apparent Diffusion Coefficient (ADC) images. Note that the white matter qCBF has been restored bilaterally (right- $21.96 \pm 8.05 \mathrm{~mL} / 100 \mathrm{~g}$-min; left $=21.52 \pm 6.98 \mathrm{~mL} / 100 \mathrm{~g}$-min) with normal ADC.

GRE-EPI sequences [9]. In both cases, $1.78 \times 1.78 \mathrm{~mm}$ voxels provide spatial resolution sufficient to distinguish white and gray matter.

\section{RESULTS}

The observed asymmetry in cerebral perfusion was quantified using a region of interest analysis. Regions of interest were placed bilaterally to cover deep white matter in the mid-ventricular region. Pre-operatively, the relative cerebral perfusion showed the right side to be $25 \%$ higher than the affected side. Post operatively, perfusion in the right and left sides were normalized to be within $3 \%$ of each other. Quantitative perfusion for the right/left hemispheres were 24.42 +/- $9.41 \mathrm{ml} / 100 \mathrm{~g}$-min and $19.53+/-5.76 \mathrm{ml} / 100 \mathrm{~g}$-min respectively (pre-operatively) and $21.96+/-8.05$ and $21.52+/-$ $6.98 \mathrm{ml} / 100 \mathrm{~g}$-min, respectively (post-operatively).

\section{DISCUSSION}

Stroke is the leading cause of disability and the third most common cause of death in the United States. Despite the various sources of thromboembolism, atherosclerosis of the carotid artery is the most common cause of ischemic stroke [10]. At the carotid bulb wall, low shear stress and flow separation with stasis and nonlaminar flow creates an environment favorable for blood-borne lipid particles to interact with the vessel wall and leads to the formation of fatty streaks containing mononuclear cells and lipid containing macrophages creating an atherosclerotic plaque [10]. On the other hand, hyperplastic fibrosclerotic changes in the subintima leads to a fibrotic thickening in the intima-media complex which hosts atherothrombotic deposition at those intimal sites leading to the development of stenosis [1]. When the deposition exceeds $1.5 \mathrm{~mm}$ it forms a plaque consisting of a fibrous cap encapsulating various cellular (inflammatory cells e.g. leukocytes and macrophages), extracellular (collagen, fibrin, and smooth muscle cells), and lipid components [1]. As the plaque develops and enlarges it increases the degree of stenosis until it becomes hemodynamically significant when exceeding $70 \%$ of the lumen thus jeopardizing CBF. However, most patients, due to the chronic nature of the disease, develop compensatory collateral supply and remain asymptomatic $[10,11]$. It is recog- nized that the presence of carotid stenosis does not predict the presence or degree of hemodynamic compromise in the distal cerebral circulation. Rather, it is the impaired cerebrovascular reactivity that is predictive of cerebral ischemia [12]. One of the goals of an operation or an intervention in patients with atherosclerotic carotid disease is to prevent ischemic stroke by improving CBF.

In patients with severe carotid stenosis, whether the mechanism of ischemia is hemodynamic or embolic may not be important [2]. Especially that, those patients with the improvement of cerebral hemodynamics after treatment will have a decreased chance of ischemic injury. "Hemodynamic impairment" is the term used to describe the presence of reduced cerebral perfusion pressure (CPP) [2]. In any region of the brain the CPP equals the mean regional arterial pressure minus either the venous pressure or the intracranial pressure, depending on which is higher [2]. Under normal circumstances CPP would be normal and the CBF would be closely matched to the resting metabolic rate of the brain tissue (Stage 0 hemodynamic impairment), consequently the Oxygen Extraction Fraction (OEF) shows little regional variation. When CPP is moderately reduced, cerebrovascular autoregulation maintains a constant CBF by arteriolar vasodilation (Stage 1 hemodynamic impairment). This may cause increase in the intravascular (Cerebral Blood Volume) CBV. With severe CPP reduction, the autoregulation fails to compensate and the $\mathrm{CBF}$ begins to decline, but the cerebral brain metabolism is maintained by the increase in OEF (Stage II hemodynamic impairment $=$ 'Misery Perfusion') $[2,13]$. The average baseline OEF is approximately $30 \%$ but may increase up to $80 \%$ [2]. Stage I hemodynamic compromise was found by some investigators to correlate with an increased stroke risk while others found no correlation [13]. A positive association of Stage II cerebral hemodynamic compromise and stroke risk has been documented $[13,14]$.

We do not routinely measure the mean arterial pressure or the CPP in brain arteries to identify hemodynamic impairment, rather we rely on identifying compensatory mechanisms by physiological imaging [2]. The main reason is that imaging modalities (such as PET and SPECT) capable of quantifying these hemodynamic variables are expensive, 
not commonly available, use ionizing radiation, and may have low spatial and temporal resolution (as is the case with SPECT) [15]. The physiologic imaging modalities in use are usually divided into three major categories. The first are those that compare the baseline measurement of blood flow or velocity to the measurement after a vasodilatory stimulus [2]. The second are those that identify autoregulatory vasodilation by either measuring the mean transit time (MTT) or calculating it from the ratio of $\mathrm{CBF}$ and $\mathrm{CBV}$ [2]. The last category of imaging modalities measure the OEF [2].

Among the commonly used physiologic imaging modalities are the Transcranial Doppler sonography (TCD) and the MR. The TCD is a noninvasive technique that has been proven reliable for measuring $\mathrm{CBF}$ velocity in large cerebral vessels [15]. This technique is not without limitations such as the variation of the measurement in the setting of cardiac arrhythmia and the inability to perform the exam on the middle cerebral artery (MCA) in 5-20\% of the patients due to the insufficient ultrasound transmission through the skull [15]. As for the perfusion-weighted MR, it is a semiquantitative technique that has a good spatial resolution and is capable of assessing the microcirculation like PET or SPECT without the need for ionizing radiation [15]. Another advantage of perfusion-weighted MRI is that it does not consume a lot more time than routine MRI. There have been some reports on the use of MRI to quantitate CBF. One example is the work by Lythgoe and colleagues [3]. This group quantitated CBF using MR, based on empirically derived scale factor. The use of empirically derived scale factors has been shown to be less reliable than patient based qCBF measurement [16]. This is particularly relevant in this case where compensatory mechanisms increase perfusion to the contralateral hemisphere.

On the other hand, another MRI technique that has the potential to determine quantitative $\mathrm{CBF}$ is arterial spin labeling (ASL). In ASL imaging, the magnetization of flowing blood is inverted upstream of the region in which the perfusion measurement is desired. As the blood flows into the imaging regions, the inverted magnetization is transferred to the tissue, altering local magnetization in proportion to $\mathrm{CBF}$. By measuring the change in local magnetization, $\mathrm{CBF}$ can be calculated. A plethora of tagging and readout schemes have been developed [17-20] and have shown the potential for measuring quantitative CBF [21-23]. The signal changes observed in ASL are small, typically 1-2\%, so that acquiring useful images requires multiple signal averages and scan times of several minutes. Furthermore, with ASL perfusion imaging, calculation of quantitative CBF depends on a variety of physiologic parameters (T1 of blood, T1 of tissue, water diffusion rate, etc) [24]. In particular, the accuracy of $\mathrm{CBF}$ depends on the time it takes for the blood to flow from the labeling region to the readout slice. Some ASL techniques can minimize this dependence when transit times are within "normal ranges". In patients with severely altered hemodynamics, this transit time can confound the accurate determination of CBF [25]. Therefore, although ASL perfusion has been shown to measure quantitative $\mathrm{CBF}$, its utility in the setting of hemodynamic compromise, where label-totissue transit times cannot be predicted, has not been established.
Conversely, the limited availability and low spatial resolution of PET perfusion scans has prompted the development of perfusion image techniques that have the potential to be disseminated in the broader medical community. MRI based qCBF images used in this case report are relatively inexpensive, safe to use and can be readily added to a comprehensive neurological MRI examination which includes: T1- and T2weighted anatomic images, angiography and diffusion images. This report uses a unique approach to quantify $\mathrm{CBF}$ using readily available MRI technology known as the "bookend technique". The bookend technique combines a singleimage two-point quantitative blood volume image with whole brain dynamic CBF images to yield whole brain quantitative $\mathrm{CBF}$ images. As with more traditional approaches that quantify perfusion, the bookend technique ultimately depends on tracer kinetics and mathematical deconvolution to calculate CBF, CBV and MTT. However unlike the commonly used MRI-based relative measures of CBF, the bookend perfusion scans are able to quantify cerebral perfusion. Furthermore, the bookend technique does not rely on empirical correction factors derived from population averaged $\mathrm{CBF}$ values. Instead, the bookend technique can determine a patient specific quantification based on the T1 changes of the parenchyma resulting form the injection of contrast agent. A large prospective study comparing the quantitative results from this technique with the relative measurements obtained from MRP and the clinical status of the patient will be needed to better estimate the value of this new technique.

\section{CONCLUSION}

MR images of relative cerebral perfusion reflect changes in local hemodynamic impairment resulting from surgical intervention. It is hoped that our ability to quantify changes in cerebral perfusion using an innovative quantitative MR will allow us to determine which patients have hemodynamically significant stenosis and would benefit from intervention. Future work involve further validation of this technique in selected patients allowing the inclusion of qCBF into the diagnostic algorithm and selection of patient who would benefit from treatment.

\section{REFERENCES}

[1] Coccheri S. Asymptomatic carotid stenosis: natural history and therapeutic implications. Pathophysiol Haemost Thromb 2003; 33(5-6): 298-301.

[2] Derdeyn CP. Cerebral hemodynamics in carotid occlusive disease. AJNR Am J Neuroradiol 2003; 24(8): 1497-9.

[3] Lythgoe DJ, Ostergaard L, William SC, et al. Quantitative perfusion imaging in carotid artery stenosis using dynamic susceptibility contrast-enhanced magnetic resonance imaging. Magn Reson Imaging 2000; 18(1): 1-11.

[4] Grubb RL, Jr., Derdeyn CP, Fritsch SM, et al. Importance of hemodynamic factors in the prognosis of symptomatic carotid occlusion. JAMA 1998; 280(12): 1055-60.

[5] Sakaie KE, Shin W, Curtin KR, McCarthy RM, Cashen TA, Carroll TJ. Method for improving the accuracy of quantitative cerebral perfusion imaging. J Magn Reson Imaging 2005; 21(5): 512-9.

[6] Shin W, Cashen TA, Horowitz SW, Sawlani R, Carroll TJ. Quantitative CBV measurement from static T1 changes in tissue and correction for intravascular water exchange. Magn Reson Med 2006; 56(1): 138-45.

[7] Rosen BR, Belliveau JW, Vevea JM, Brady TJ. Perfusion imaging with NMR contrast agents. Magn Reson Med 1990; 14(2): 249-65.

[8] Ostergaard L, Sorensen AG, Kwong KK, Weisskoff RM, Gyldensted C, Rosen BR. High resolution measurement of cerebral blood flow using intravascular tracer bolus passages. Part II: Experimen- 
tal comparison and preliminary results. Magn Reson Med 1996; 36(5): 726-36

[9] Sorensen AG, Reimer P. Cerebral MR Perfusion Imaging: Principles and current applications. Stuttgart: Thieme; 2000.

[10] Allain R, Marone LK, Meltzer J, Jeyabalan G. Carotid endarterectomy. Int Anesthesiol Clin 2005; 43(1): 15-38.

[11] Giannoukas AD, Labropoulos N, Smith FC, Venables GS, Beard JD. Management of the near total internal carotid artery occlusion. Eur J Vasc Endovasc Surg 2005; 29(3): 250-5.

[12] Kamouchi M, Kishikawa K, Okada Y, Inoue T, Ibayashi S, Iida M. Poststenotic flow and intracranial hemodynamics in patients with carotid stenosis: transoral carotid ultrasonography study. AJNR Am J Neuroradiol 2005; 26(1): 76-81.

[13] Grubb RL, Jr. Extracranial-intracranial arterial bypass for treatment of occlusion of the internal carotid artery. Curr Neurol Neurosci Rep 2004; 4(1): 23-30.

[14] Herzig R, Hlustik P, Urbanek K, et al. Can we identify patients with carotid occlusion who would benefit from EC/IC bypass? Review. Biomed Pap Med Fac Univ Palacky Olomouc Czech Repub 2004; 148(2): 119-22.

[15] Doerfler A, Eckstein HH, Eichbaum M, et al. Perfusion-weighted magnetic resonance imaging in patients with carotid artery disease before and after carotid endarterectomy. J Vasc Surg 2001; 34(4): 587-93.

[16] Mukherjee P, Kang HC, Videen TO, McKinstry RC, Powers WJ, Derdeyn CP. Measurement of cerebral blood flow in chronic carotid occlusive disease: comparison of dynamic susceptibility contrast perfusion MR imaging with positron emission tomography. AJNR Am J Neuroradiol 2003; 24(5): 862-71.
[17] Alsop DC, Detre JA. Multisection cerebral blood flow MR imaging with continuous arterial spin labeling. Radiology 1998; 208(2): 410-6.

[18] Detre JA, Zhang W, Roberts DA, et al. Tissue specific perfusion imaging using arterial spin labeling. NMR Biomed 1994; 7(1-2): $75-82$.

[19] Edelman RR, Chen Q. EPISTAR MRI: multislice mapping of cerebral blood flow. Magn Reson Med 1998; 40(6): 800-5.

[20] Golay X, Hendrikse J, Lim TC. Perfusion imaging using arterial spin labeling. Top Magn Reson Imaging 2004; 15(1): 10-27.

[21] Roberts DA, Detre JA, Bolinger L, Insko EK, Leigh JS, Jr. Quantitative magnetic resonance imaging of human brain perfusion at 1.5 $\mathrm{T}$ using steady-state inversion of arterial water. Proc Natl Acad Sci USA 1994; 91(1): 33-7.

[22] Wong EC, Buxton RB, Frank LR. Quantitative imaging of perfusion using a single subtraction (QUIPSS and QUIPSS II). Magn Reson Med 1998; 39(5): 702-8.

[23] Wong EC, Buxton RB, Frank LR. Quantitative perfusion imaging using arterial spin labeling. Neuroimaging Clin North Am 1999; 9(2): 333-42.

[24] Alsop DC, Detre JA. Reduced transit-time sensitivity in noninvasive magnetic resonance imaging of human cerebral blood flow. J Cereb Blood Flow Metab 1996; 16(6): 1236-49.

[25] Chalela JA, Alsop DC, Gonzalez-Atavales JB, Maldjian JA, Kasner SE, Detre JA. Magnetic resonance perfusion imaging in acute ischemic stroke using continuous arterial spin labeling. Stroke 2000; 31(3): 680-7.

(C) Adel et al.; Licensee Bentham Open

This is an open access article licensed under the terms of the Creative Commons Attribution Non-Commercial License (http://creativecommons.org/licenses/by-nc/3.0/) which permits unrestricted, non-commercial use, distribution and reproduction in any medium, provided the work is properly cited. 